\title{
NÍVEL DE ANSIEDADE DE CLIENTES SUBMETIDOS A CINEANGIOCORONARIOGRAFIA E DE SEUS ACOMPANHANTES ${ }^{1}$
}

\author{
Eliane da Silva Grazziano \\ Estela Regina Ferraz Bianchi ${ }^{3}$
}

Grazziano ES, Bianchi ERF. Nível de ansiedade de clientes submetidos a cineangiocoronariografia e de seus acompanhantes. Rev Latino-am Enfermagem 2004 março-abril; 12(2):168-74.

Este estudo exploratório e com abordagem quantitativa teve como objetivos identificar o nível de ansiedade de 40 acompanhantes e 40 clientes submetidos a cineangiocoronariografia pela primeira vez, no período de espera do exame, e correlacionar os dados entre si e com as variáveis sóciodemográficas, utilizando a Escala de Ansiedade-Estado (IDATE). Concluiu-se que a maioria dos clientes apresenta baixo nível de ansiedade e a maioria dos acompanhantes apresenta nível médio de ansiedade. Na análise das correlações, não houve relação entre o nível de ansiedade e as variáveis sóciodemográficas da população estudada. Na análise das comparações, observou-se que o acompanhante do sexo masculino é mais ansioso que aquele do sexo feminino. As intervenções de enfermagem, nesse período, podem ser mais eficazes se incluírem ações para redução da ansiedade dos familiares/acompanhantes.

DESCRITORES: cuidados de enfermagem; ansiedade; cuidadores; cateterismo cardíaco

\section{CAREGIVERS AND PATIENT'S ANXIETY LEVEL DURING CARDIAC CATHETERIZATHION}

This exploratory and quantitative study aimed to identify the anxiety level of 40 caregivers and 40 patients before cardiac catheterization and to establish a correlation among them and with sociodemographic variables, by means of the Anxiety-State Scale (IDATE). It was concluded that most of the patients present low anxiety levels, while most caregivers demonstrate medium levels of anxiety. No statistical correlation was found between anxiety levels and sociodemographic variables in the correlation analysis. In a comparative analysis, we observed that male caregivers are more anxious than female ones. Nursing interventions during this period can be more efficient if they include actions to reduce anxiety among family members/caregivers.

DESCRIPTORS: nursing care; anxiety; caregivers; cardiac catheterization

\section{NIVEL DE ANSIEDAD DE ACOMPAÑANTES Y CLIENTES DURANTE EL CATETERISMO CARDÍACO}

Este estudio exploratorio de carácter cuantitativo que tuvo como objetivo analizar el nivel de ansiedad de 40 acompañantes y 40 clientes sometidos por primera vez al cateterismo cardíaco y durante el periodo de espera del examen, con aplicación de la Escala de Ansiedad-Estado (IDATE). La conclusión es que la mayoría de los clientes tiene bajo nivel de ansiedad e sus acompañantes tienen nivel medio de ansiedad. Pudimos verificar en el análisis estadístico que no hay relación entre el nivel de ansiedad y la variables sociodemográficas de la población estudiada. El análisis de las comparaciones muestra que el acompañante del sexo masculino es más ansioso que el femenino. Intervenciones específicas de enfermería para reducir ansiedad del acompañante durante el cateterismo cardíaco podrán ayudar con la ansiedad de los clientes.

DESCRIPTORES: atención de enfermería; ansiedad; cuidadores; cateterismo

\footnotetext{
${ }^{1}$ Resumo de Dissertação de Mestrado apresentado à Escola de Enfermagem da Universidade de São Paulo; ${ }^{2}$ Enfermeira, Docente da Faculdade Santa Marcelina, e-mail: elisg@terra.com.br; ${ }^{3}$ Enfermeira, Professor Livre-Docente da Escola de Enfermagem da Universidade de São Paulo, email: erfbianc@usp.br
} 
Várias são as expectativas, sentimentos e emoções envolvidas no ato de "esperar" por algum acontecimento ou por alguém. Em situações de hospitalização, tratamentos médicos e exames diagnósticos, o período de espera pode tornar-se angustiante, podendo levar ao stress e à ansiedade ${ }^{(1)}$.

Estudos demonstram que clientes agendados para qualquer exame diagnóstico invasivo apresentam as mesmas inquietações: ansiedade relacionada à ameaça da integridade física e ao bem-estar, como também às implicações advindas do resultado diagnóstico ${ }^{(2)}$. A família é um sistema composto por indivíduos, no qual processos de doenças e de adaptação são experimentados por todos. Durante a hospitalização, em procedimentos terapêuticos, cirúrgicos ou diagnósticos, o sistema familiar sente-se ameaçado por vários fatores como a mudança de papéis familiares, a incerteza do prognóstico e quebra de rotina, o que leva à precipitação de crises, mesmo em famílias bem estruturadas, em que ambos, família e cliente, são afetados pelo stress nessa condição $0^{(1,3)}$.

Durante atuação em laboratório de hemodinâmica, fui solicitada várias vezes a prestar atendimento em caráter emergencial a acompanhantes que apresentaram malestar súbito, devido a crises hipertensivas no período de espera para a realização de cineangiocoronariografias (CAT).

O CAT é uma técnica diagnóstica que permite confirmar a presença de disfunção clínica, sugerida por outros métodos diagnósticos indicativos de infarto do miocárdio ou angina instável, e para determinar as condições associadas presentes.

Embora seja um procedimento invasivo, o CAT pode ser realizado em um ambiente ambulatorial exigindo preparo prévio e um período de observação pós-exame de quatro a oito horas, dependendo da via de acesso radial ou femoral, respectivamente. Durante o exame, podem ocorrer complicações, tais como: arritmias cardíacas, infartos do miocárdio, paradas cardiorrespiratórias ou rompimentos de artérias, devido à manipulação de vasos.

A dinâmica executada na maioria dos laboratórios de hemodinâmica dificulta o contato prévio do enfermeiro com o cliente e seus familiares, sendo que o enfermeiro, em muitos serviços, nem chega a conhecer o(s) acompanhante(s) de seus clientes. Devido ao tempo relativamente curto de intervenção, em média 20 minutos, somente alguns dados relevantes à execução do exame são coletados pelo auxiliar ou técnico de enfermagem, tais como: sinais vitais, peso e altura e alergia a iodo. Esse quadro pode mudar quando o cliente encontra-se internado na instituição que realizará o exame ou quando é feito em instituições cuja assistência de enfermagem já está sistematizada.

Em alguns serviços, o familiar permanece com o cliente em uma ante-sala, até à entrada desse no laboratório de exames. Porém, na grande maioria, somente o cliente é admitido em área anexa ao laboratório onde pode permanecer de 30 minutos até duas horas, aguardando o início do procedimento, sem que o acompanhante seja informado dessa espera.

Nessas ocasiões o familiar se vê, de um momento para outro, responsável não somente em fornecer suporte emocional ao cliente, frente à constatação de uma coronariopatia, com vários graus de risco, como também em agilizar trâmites burocráticos necessários para uma internação de urgência (incluindo despesas médicas e hospitalares) e ser fonte de informação ao restante da família.

Devido à especificidade do CAT, dados como alergias a iodo, hipertensão e uso de hipoglicemiantes orais são pesquisados pela equipe que atende o cliente. Tanto os clientes quanto os acompanhantes devem ser informados sobre os riscos inerentes ao procedimento e consentirem com a realização do mesmo, assinando um consentimento pós-informado.

Em minhas observações, notei que esse procedimento de coleta de dados e a solicitação do consentimento pós-informado aos acompanhantes levavam a manifestações como tremores na voz, palidez, uma leve agitação, questionamentos através do "olhar" e uma "tempestade" de perguntas era realizada, abordando desde a segurança do procedimento a uma possível internação do cliente.

Refletindo sobre essa situação, passei a questionar: quais seriam os fatores que estariam atuando no comportamento dos acompanhantes? A ansiedade poderia estar interferindo no seu comportamento? Em caso positivo, em que nível? Haveria relação entre o grau ou nível de ansiedade do cliente e do acompanhante? O nível de ansiedade do acompanhante é maior que a do cliente?

Considerando os riscos envolvidos, o tempo de espera relativamente longo se comparado à duração do próprio procedimento, o sentimento de exclusão do 
acompanhante frente à separação entre ele e o cliente, muitas vezes por um período de tempo superior ao esperado, e a inexistência de rotinas de enfermagem específicas que ofereçam algum suporte ao familiar/ acompanhante, pode-se supor que estados emocionais permeiem as relações enfermeiro/cliente/acompanhante e que o stress esteja presente.

\section{STRESS E ANSIEDADE}

O stress é definido como um conjunto de manifestações fisiológicas e emocionais em resposta a estímulos estressores e que obedece a uma seqüência de acontecimentos mais ou menos constante, onde ocorrem simultaneamente: estimulação adrenal, diminuição do timo, ulcerações gastrintestinais, perda de peso, alterações na composição química do corpo, entre outras $^{(4)}$. Como estímulos estressores encontram-se eventos que podem acometer uma única pessoa ou um pequeno grupo de pessoas, tais como: morte, doenças crônicas ou incapacitantes, divórcio e até mesmo o nascimento ${ }^{(5)}$.

O stress manifesta-se quando existem demandas para o indivíduo que sobrecarregam ou excedem seus recursos adaptativos, sendo a maneira de enfrentamento o fator que irá predizer a intensidade de suas respostas ${ }^{(6)}$.

Os sintomas de stress na área emocional vão desde a apatia até à depressão, com nuances de desânimo e sensação de desalento, hipersensibilidade emotiva, até raiva, ira, irritabilidade e ansiedade, além de ter potencial para desencadear surtos psicóticos e crises neuróticas ${ }^{(7)}$. A interpretação de um estímulo interno ou externo como ameaçador ou perigoso desencadeará uma reação emocional que é caracterizada por estado de ansiedade.

A ansiedade primária é conceituada como um fenômeno adaptativo necessário ao homem para enfrentamento das situações cotidianas, com duração e intensidade que variam de indivíduo para indivíduo e de acordo com as diferentes situações ${ }^{(8)}$. Caracteriza-se por um conjunto de manifestações físicas, como taquicardia, sudorese, hiperventilação e tensão muscular, e psicológicas evidenciadas por apreensão, alerta e inquietude, mediados pelos sistemas gabaérgico, serotonérgico, dopaminérgico, neuropeptidérgicos, entre outros $^{(9)}$.

O ambiente externo possui fatores estressantes quando exige do indivíduo uma adaptação. $\mathrm{O}$ ambiente hospitalar, com suas regras estabelecidas, obriga a sua comunidade (funcionários, clientes, médicos, familiares) a adaptar-se às situações geradoras de ansiedade. $\mathrm{O}$ convívio com a doença, sofrimento e morte leva à constante adaptação dos profissionais e familiares que dividem esse ambiente.

No que se refere ao período de espera, durante um procedimento diagnóstico, pode-se supor que essa situação e a antecipação do resultado do exame provocarão sentimentos e repercussões psicofísicas que, consideradas as diferenças individuais, levarão a comportamentos de ajuste com finalidade de enfrentamento da situação. Dessa forma, tanto o cliente quanto o acompanhante manifestam estados emocionais de ansiedade. No presente estudo será analisada a ansiedade adaptativa ou primária e não a patológica, ocasionada por distúrbios mentais.

Em minha vivência como profissional de enfermagem, atuante em laboratório de hemodinâmica, observei que os acompanhantes manifestavam estados emocionais de ansiedade com maior freqüência e com maior intensidade que os próprios clientes, fato inquietador haja vista a inexistência de intervenções de enfermagem específicas direcionadas aos acompanhantes.

Esses fatores colaboravam para tornar árida a comunicação entre equipe multiprofissional e acompanhantes dos clientes. Para que ocorram interações enfermeiro/cliente/acompanhante satisfatórias, os níveis de ansiedade dos acompanhantes devem ser diminuídos por meio de intervenções programadas para esse fim.

Considerando a ausência de estudos que analisem a ansiedade dos acompanhantes de clientes submetidos a exames diagnósticos em geral e, em particular, a cineangiocoronariografia, este estudo propõe-se a analisar o nível de ansiedade do acompanhante e do cliente e sua correlação com as variáveis sóciodemográficas.

\section{OBJETIVOS}

O presente estudo teve por objetivos:

- caracterizar a população estudada de acordo com as variáveis sexo, faixa etária, procedência e grau de escolaridade;

- identificar o nível de ansiedade do indivíduo submetido a cineangiocoronariografia (CAT) e de seu acompanhante; - correlacionar o nível de ansiedade dos acompanhantes e dos clientes entre si e com as variáveis sóciodemográficas do estudo. 
METODOLOGIA

Este é um estudo exploratório, descritivo, correlacional, comparativo, com abordagem quantitativa, realizado com 40 acompanhantes/familiares e 40 clientes submetidos ao procedimento de cateterismo coronariano (CAT), em um Laboratório de Hemodinâmica de um hospital geral, de grande porte, localizado no município de São Paulo. O setor possui sete salas destinadas ao diagnóstico e terapêutica das afecções coronarianas e cardíacas, realizando aproximadamente 100 procedimentos/dia a clientes particulares, conveniados e do SUS. A amostra foi aleatória e determinada por análise estatística.

Após a aprovação pelo Comitê de Ética e Pesquisa da Instituição procedeu-se à coleta de dados por meio de entrevista individual, momentos antes do início dos exames, segundo as seguintes condições de seleção e inclusão:

A. quanto ao cliente: indivíduos adultos, com mais de 18 anos, submetidos ao CAT eletivo, pela primeira vez; alfabetizados e que concordaram em participar da pesquisa;

B. quanto ao acompanhante: indivíduos adultos, com mais de 18 anos, alfabetizados, sem restrição de vínculo afetivo e que concordaram em participar da pesquisa.

O Instrumento utilizado foi composto de duas partes, a saber: questionário para caracterização do familiar e do cliente, contendo informações de identificação, grau de escolaridade e grau de parentesco, e pelo Inventário de Ansiedade Traço-Estado - IDATE ${ }^{(8)}$. Esse inventário é composto por duas escalas distintas de autorelatório com 20 afirmações cada uma, utilizada para medir dois conceitos distintos de ansiedade: estado de ansiedade (A-estado) e traço de ansiedade (A-traço).

Neste estudo foi utilizada a escala de A-estado visto que essa escala foi concebida para análise do estado emocional transitório, caracterizado por sentimentos de apreensão e tensão conscientemente percebidos, e pelo aumento na atividade do sistema nervoso autônomo. Os pesos dos itens em que os escores indicam alta ansiedade são iguais aos números escolhidos pelo respondente no formulário de teste. Para os itens em que escores altos indicam baixa ansiedade, os pesos são invertidos. Os pesos de respostas marcadas 1, 2, 3 e 4 para os itens a inverter são 4, 3, 2 e 1, respectivamente. Os itens invertidos da subescala A-estado, são: 1, 2, 5, 8, 10, 11, 15, 16, 19 e 20 .

Tendo em vista que, em seu formato original, o instrumento apresentou um alfa de Cronbach muito baixo (inferior a 0,18), para a população-alvo, excluiu-se o item que estava causando tal inconsistência, a saber, o item 11 , o que elevou o alfa para um valor superior a 0,50. A amplitude de escores possíveis para o instrumento utilizado variou de um mínimo de 19 a um máximo de 76 e os níveis de ansiedade foram categorizados como se segue: de 19 a 40 pontos - Baixo; de 41 a 60 pontos Médio e de 61 a 76 pontos - Alto.

A análise descritiva foi feita sobre as variáveis respostas e as variáveis sóciodemográficas dos clientes e familiares utilizando distribuições de freqüências e as medidas resumo média, desvio padrão, mediana, mínimo e máximo na produção de estimativas pontuais e intervalos de confiança de $95 \%$ na produção de estimativas intervalares.

\section{RESULTADOS}

Os resultados obtidos no presente estudo demonstraram que houve predominância de clientes do sexo feminino (21-52,5\%), com idade entre 61 e 70 anos (17-42,5\%), vindos do interior do Estado de São Paulo (24-60\%) e com 0 a 04 anos de escolaridade, isto é, ensino fundamental incompleto (21-52,5\%).

Em relação ao perfil dos acompanhantes, houve predominância do sexo feminino (30-75\%), com idade entre 31 e 40 anos (13-32,5\%), vindos do interior do Estado de São Paulo (22-55\%), com 11 a 14 anos de escolaridade, isto é, ensino médio completo ou superior incompleto (11$27,5 \%$ ) e categorizados como filhos (17-42,5\%).

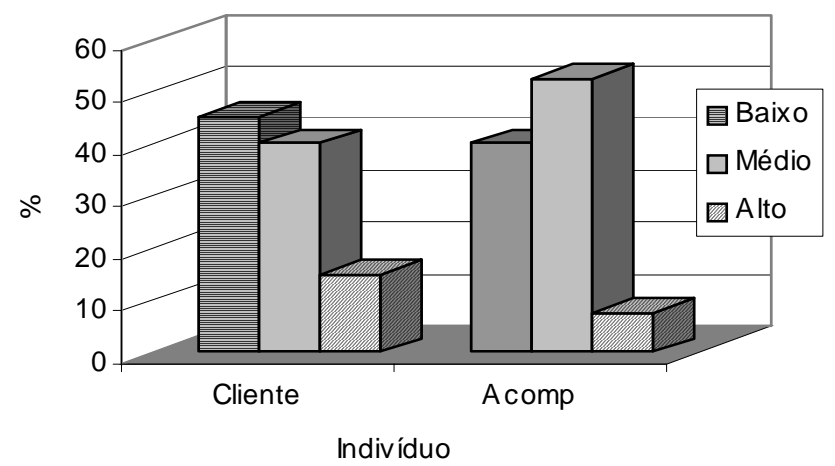

Figura 1 - Distribuição percentual do nível de ansiedade dos 40 clientes e dos 40 acompanhantes. São Paulo, 2001 
$\mathrm{Na}$ análise do nível de ansiedade do indivíduo submetido ao CAT, os resultados demonstraram que houve prevalência de baixo nível de ansiedade (18-45\%); 06 (15\%) clientes apresentaram nível alto de ansiedade e 16 (40\%) apresentaram nível médio de ansiedade.

Os familiares dos clientes apresentaram, em sua maioria, nível médio de ansiedade (21-52,5\%); 03 (7,5\%) acompanhantes apresentaram nível alto de ansiedade e 16 (40\%) apresentaram nível baixo de ansiedade (Figura 1).

A análise dos dados demonstrou a inexistência de diferença estatisticamente significativa entre os escores médios de ansiedade dos clientes e acompanhantes. Entretanto, houve correlação positiva entre o nível de ansiedade do acompanhante e a variável "sexo", isto é, há evidência estatisticamente significativa de que o familiar do sexo masculino apresenta nível de ansiedade superior ao do familiar do sexo feminino (Tabela 1).

Tabela 1 - Correlação entre o nível de ansiedade dos indivíduos e as variáveis ordinais ${ }^{\mathrm{a}}$ e variáveis nominais dicotômicas $^{\text {b }}$ do estudo. São Paulo, 2001

\begin{tabular}{ccc}
\hline & NAC & NAA \\
\hline Cliente do sexo masculino & 0,08 & 0,17 \\
Faixa etária do cliente & 0,24 & $-0,02$ \\
Grau de instrução do cliente & $-0,12$ & 0,23 \\
& & \\
Acomp. do sexo masculino & $-0,13$ & $0,28^{*}$ \\
Faixa etária do acomp. & 0,19 & $-0,04$ \\
Grau de instrução do acomp. & 0,19 & $-0,11$ \\
Grau de parentesco & 0,19 & 0,13 \\
NA do cliente (NAC) & 1,00 & $-0,15$ \\
NA do acompanhante (NAA) & $-0,15$ & 1,00 \\
\hline
\end{tabular}

( $\left.{ }^{a}\right)$ A Correlação foi avaliada pelo Coef. de Correlação Ordinal de Spearman

$\left.{ }^{b}\right)$ A Correlação foi avaliada pelo Coef. de Correlação de Pearson

$\left(^{*}\right)$ Correlação estatisticamente significativa $(p<0,05)$

Verificou-se, também, a inexistência de correlação do nível de ansiedade com as variáveis: faixa etária, grau de instrução e grau de parentesco, como também não foi observada diferença estatisticamente significativa entre o nível de ansiedade dos indivíduos quanto à procedência ou o grau de instrução.

\section{DISCUSSÃO}

A predominância de clientes do sexo feminino, neste estudo, reitera o aumento da incidência de morbidade por doenças cardiovasculares na população feminina e em idades mais precoces. A mortalidade pelas doenças cardíacas no Brasil é das mais elevadas, quando comparadas a outros países e, ao contrário desses, a taxa de óbito por doença isquêmica do coração, causada pela aterosclerose, na população feminina, em quatro capitais brasileiras (São Paulo, Rio de Janeiro, Curitiba e Porto Alegre) superam, praticamente, as taxas de todos os países desenvolvidos escolhidos para comparação em estudos recentes ${ }^{(10)}$.

Os dados coletados corroboram a predominância de familiares do sexo feminino, reforçando os dados conhecidos em pesquisas anteriores ${ }^{(3,11)}$.

Com relação ao grau de parentesco, nota-se sutil predominância de indivíduos categorizados como filhos (42,5\%) em relação aos cônjuges $(37,5 \%)$, dados também obtidos por outros pesquisadores ${ }^{(12)}$. Tal achado justificase pelo fato de haver predominância de clientes com idade entre 61 e 70 anos (17-42,5\%), dado que reforça a inversão dos papéis entre pais e filhos, necessitando de maior apoio dos filhos, pela idade avançada dos pais.

O estudo demonstrou que, a maioria dos clientes (24-60\%) e dos familiares (22-55\%) veio do interior do Estado. Pesquisas realizadas no Brasil demonstram que quanto menor o nível de escolaridade e condição sócioeconômica, maior é a exposição aos fatores de risco para doenças cardiovasculares, entre eles a hipertensão, o tabagismo, o alcoolismo, a obesidade e o sedentarismo, elevando a incidência dessas moléstias em regiões fora da área urbana. Os fatores ambientais, a baixa renda familiar, o baixo nível educacional, a atividade profissional, exposição ao stress, sedentarismo, falta de lazer, sono e repouso, excesso de peso e vínculo familiar influenciam na manutenção dos fatores de risco ${ }^{(12)}$.

Os dados obtidos confirmam a afirmação anterior, tendo em vista o baixo grau de escolaridade dos clientes, situação que freqüentemente está associada ao baixo nível sócioeconômico.

$\mathrm{Na}$ análise do nível de ansiedade, o estudo demonstrou predominância de baixo nível de ansiedade no grupo de clientes (18-45\%) e de médio nível de ansiedade no grupo de familiares (21-52,5\%). O escore médio para a ansiedade dos clientes é de 42,75 ( $\mathrm{DP}=10,17)$; a mediana é de 41 com variação de 25 a 68, enquanto que o escore médio para a ansiedade dos acompanhantes é 42,85 (DP=9,91), a mediana é de 44,5 com variação de 23 a 64 . 
Os dados obtidos foram similares àqueles de estudos anteriores que demonstraram que clientes submetidos ao CAT apresentavam-se ansiosos antes do procedimento e manifestavam preocupação com relação ao tempo de espera, à técnica utilizada, a possíveis complicações, à necessidade de cirurgia e ao tempo de recuperação(2).

O nível de ansiedade dos clientes, verificado em nosso estudo, pode ser considerado dentro do esperado já que o escore de ansiedade normal para um adulto, segundo o IDATE, varia de 37 a 40 . Uma possível justificativa para esse achado estaria apoiada no fato do procedimento ser realizado em ambiente ambulatorial e ter curta duração, gerando assim menos expectativa do que aquela que poderia ocorrer em uma internação hospitalar. Além disso, muitos clientes verbalizaram sua confiança na instituição e no desempenho dos médicos hemodinamicistas, indicando ser esse um fator importante para que se sentissem um pouco mais tranqüilos.

$\mathrm{Na}$ análise do nível de ansiedade familiar, houve predominância de nível médio de ansiedade (21-52,5\%). Como não há conhecimento de estudos realizados com acompanhantes durante a espera de realização de exames diagnósticos, ao se comparar os achados deste estudo com os identificados na literatura, verifica-se que o nível de ansiedade obtido é similar aos encontrados em pesquisas anteriores relacionadas a períodos de espera para procedimentos cirúrgicos e durante internações em unidades de terapia intensiva ${ }^{(13-14)}$.

A busca por informações pelos familiares foi uma constante durante a coleta de dados. Pode-se supor que essa atitude seja o reflexo de estratégia de enfrentamento disponível, tentando obter informações que tragam bemestar e diminuam a sensação de exclusão durante esse período $^{(13,15)}$.

Os indivíduos da população estudada foram unânimes em manifestar que a separação entre eles, durante o período de espera para a realização do exame, foi um dos fatores que os tornavam mais preocupados e ansiosos. Somente um dos familiares, portador de hipertensão arterial, referiu mal-estar, sendo encaminhado ao pronto atendimento, permanecendo em repouso por algumas horas.

Os resultados obtidos confirmaram as observações iniciais que indicavam que tanto o cliente quanto o familiar apresentam-se ansiosos na fase que antecede a realização do CAT, e confirma a hipótese inicial de que o familiar apresenta nível de ansiedade maior que o do cliente. A constatação de que o nível de ansiedade é maior para os acompanhantes do sexo masculino causa surpresa, já que em vários estudos as mulheres são consideradas mais ansiosas que os homens.

A inexistência de diferença estatisticamente significativa entre o nível de ansiedade dos indivíduos quanto ao sexo, procedência ou grau de instrução é similar ao encontrado na literatura que indica não haver relação significativa do nível de ansiedade dos clientes submetidos ao CAT relacionada à idade, sexo ou grau de instrução ${ }^{(16)}$.

Sendo a ansiedade caracterizada por um conjunto de manifestações físicas, tais como taquicardia, sudorese, hiperventilação e tensão muscular, e psicológicas, evidenciadas por apreensão, alerta e inquietude, pode-se supor que o seu controle durante a realização do CAT é de grande importância, pois o cliente menos ansioso colabora, mantendo-se em um posicionamento adequado na mesa de exames, apresentando menos reações vagais indesejáveis e mantendo sinais vitais dentro de parâmetros seguros.

Considerando que qualquer mudança que afete um dos membros de uma família, também afetará um outro membro ou todo o sistema familiar, as intervenções direcionadas para a família também repercutirão em cada um de seus membros. Em outras palavras, o enfermeiro pode reduzir o nível de ansiedade do cliente, promovendo a redução do nível de ansiedade dos familiares.

A utilização de estratégias que forneçam informação sistematizada ao familiar poderá possibilitar participação mais efetiva na recuperação do cliente, além de melhorar a qualidade das relações entre a equipe de enfermagem e familiares, tradicionalmente consideradas como problemáticas ${ }^{(13,17-18)}$.

Tal interação pode parecer mais facilmente obtida quando há um espaço de tempo que a permita, como o que acontece durante períodos de espera em uma internação hospitalar. Porém, como efetivar essa interação durante procedimentos diagnósticos invasivos relativamente curtos tal qual ocorre no CAT? Para responder esta indagação, reporto-me a algumas perguntas feitas por quase a totalidade dos indivíduos que participaram deste estudo: por que não posso ficar perto do meu familiar até ele ser chamado para a sala de exames? Por que temos que chegar com tantas horas de antecedência e permanecer esperando por tanto tempo? Como vai ser o exame?

Diante dos resultados obtidos pode-se tecer 
sugestões de intervenções de enfermagem na assistência ao familiar, nesse período, tais como:

- ampliação do conhecimento, pelo enfermeiro, do impacto emocional causado pelo processo de doença, hospitalização e execução de exames invasivos, tal como o CAT, no sistema familiar e em seus membros;

- elaboração de estratégias que melhorem a qualidade da comunicação entre enfermeiros e familiares, considerando as alterações emocionais que permeiam essas relações e a dinâmica da unidade. Por exemplo: otimizando o fluxo de informações durante o agendamento do exame ou através de folhetos explicativos, orientações sobre o preparo, realização e recuperação pós CAT; durante a recepção do cliente na unidade, através de filmes explicativos; diminuindo o período de espera através da distribuição racional dos horários de exames; mantendo

\section{REFERÊNCIAS BIBLIOGRÁFICAS}

1. Romano BW. A família e o adoecer durante a hospitalização. Rev Soc Cardiol Estado São Paulo 1997; 7(5 Supl A):58-62.

2. Hartfield MT, Cason CL, Cason GC. Effects of information about a threatening procedure on patient' expectations and emotional distress. Nurs Res 1981; 31:202-6.

3. Koller PA. Family needs and coping strategies during illness crisis. Clin Issues Crit Care Nurs 1991; 2(2):338-45.

4. Selye H. The Stress of life. 4th ed. New York: McGraw-Hill Book; 1956.

5. Lazarus RS, Folkman S. Stress: appraisal and coping. New York: Springer; 1984. p. 22-54.

6. Peniche ACG. A influência da ansiedade na resposta do cliente no período pós-operatório imediato. [tese]. São Paulo (SP): Escola de Enfermagem/USP; 1998.

7. Lipp MEN, editor. Stress: conceitos básicos. Campinas (SP): Papirus; 1996.

8. Spielberger $C D$, Gorsuch RL, Lushene RE. Inventário de ansiedade traço-estado - IDATE. Trad. de Ângela M. B. Biaggio e Luiz Natalício. Rio de Janeiro (RJ): CEPA; 1979.

9. Dractu L, Lader M. Ansiedade: conceito, classificação e biologia - uma interpretação contemporânea da literatura. J Bras Psiquiatr 1993; 42(1):19-32.

10. Laurenti R, Buchalla CM. Myths about cardiovascular diseases. Arq Bras Cardiol 2001; 76(2):99-104.

11. Faro ACM. Aspectos teóricos sobre a família em um contexto histórico e social. Nursing 2000; 3(3):26-28. (edição brasileira)

12. Ferreira BA. Fatores de risco para doença coronariana: comportamentos e percepções de clientes pós-intervenção hemodinâmica. [dissertação]. São Paulo (SP): Escola de Enfermagem/ USP; 1999.

13. Leske JS. Anxiety of elective surgical patient's family members - relationship between anxiety levels and family characteristics. AORN J 1993; 57(5):1091-103. o familiar informado sobre atrasos no início do exame; manutenção do familiar próximo ao cliente até o momento desse ser encaminhado à sala de exame, e adequação da sala de espera do laboratório de hemodinâmica para que permita possibilidades de informação e distração durante esse período;

- a implantação da SAE em um modelo específico para o Laboratório de Hemodinâmica, incluindo intervenções para o familiar e a conscientização e disponibilidade da essência do papel do enfermeiro como interlocutor entre a ciência e a humanização do cuidado.

O papel do enfermeiro na humanização do cuidado é de suma importância e espera-se que este estudo tenha contribuído para uma reflexão sobre o tema e como estímulo para o desenvolvimento de novas pesquisas que permitam a generalização dos dados apresentados.

14. Leske JS. Intraoperative progress reports decrease family member's anxiety AORN J 1996; 64(3):424-36.

15. Trimm D. Spousal coping during the surgical wait Perianesth Nurs 1997; 12(3):141-51.

16. Peterson M. Patient anxiety before cardiac catheterization: an inventory study. Heart Lung 1991; 20:643-7.

17. Fowlie P, Francis $H$, Russel S. A perioperative communication with families. Can Nurs 2000; 96(8):30-3.

18. Siqueira MM, Watanabe FS, Ventola A. Desgastes físico e mental de auxiliares de enfermagem: uma análise sob o enfoque gerencial. Rev Latino-am Enfermagem 1995 jan; $3(1): 45-57$. 